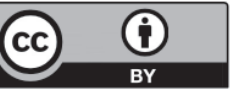

https://creativecommons.org/licenses/by/4.0/

\title{
RETOS DEL CAPITAL HUMANO DE LAS PYMES DE BOGOTÁ Y SU IMPACTO EN LA INNOVACIÓN Y COMPETITIVIDAD
}

\author{
The challenges of the human capital on SMES in Bogotá \\ and their impact on innovation and competitiveness
}

RICARDO VILLALBA, HAROLD DAVID TORRES ${ }^{2}$

Recibido:02 de noviembre de 2018. Aceptado:12 de diciembre de 2018

DOI: http://dx.doi.org/10.21017/rimci.2019.v6.n11.a58

\begin{abstract}
Resumen
El presente artículo se realizó con el fin de identificar las principales problemáticas que hoy provocan que las pymes prestadoras de servicio de la ciudad de Bogotá tengan dificultades para aprovechar las oportunidades que se presentan en el mercado, analizar los factores que impiden su crecimiento y estabilidad; desde el punto de vista de la cadena de suministro, buscando una solución interna en las organizaciones usando para esta labor el capital humano. Haciendo una revisión bibliográfica se busca identificar las problemáticas que afrontan las pymes de la ciudad de Bogotá y sus posibles soluciones para aumentar la competitividad.
\end{abstract}

Palabras clave: pymes, cadena de suministro, capital humano, competitividad.

\begin{abstract}
The present article made with the end to identify the main problematic that today cause that the pymes providers of service of the city of Bogota have difficulties to take advantage of the opportunities that present in the market, analyze the factors that prevent his growth and stability; From the point of view of the chain of supply, looking for an internal solution in the organizations using for this work the human capital. Doing a bibliographic review looks for identify the problematic that face the pymes of the city of Bogota and his viable solutions to increase the competitiveness.
\end{abstract}

Keywords: pymes, supply chain management, human capital, Competitiveness.

\section{INTRODUCCIÓN}

LAS PYMES en Colombia se han constituido como la principal fuente de empleo del país y según el fondo monetario internacional lo seguirán siendo por los próximos 25 años dado el interés de los emprendedores por generar empresa y la gran cantidad de existencias de estas en el país, así mismo se han transformado en uno de los principales ejes de estudio e investigación.
Las investigaciones cuyo tema principal son las pymes recaen en temas de perspectiva económica y gestión donde las problemáticas significativas detectadas son:

- Falta de crédito para capacitaciones.

- Gestión administrativa deficiente.

- Falta de asociación.

- Falta de apoyo gubernamental.

1 Docente Investigador del Grupo de Investigación OCA Operaciones Calidad Administración - Línea de investigación Diseño y gestión de la cadena de suministro de la Corporación Universitaria Republicana. Ingeniero de Producción, Universidad EAN, Especialista en Gerencia de Proyectos, Universidad EAN, actualmente cursando Maestría en Administración de Empresas, Universidad EAN. Correo electrónico: ricardoavillalbar@gmail.com

2 Estudiante de Ingeniería Industrial Semillero de Investigación de la Corporación Universitaria Republicana. 
Para evidenciar las problemáticas que afectan a las pymes de Bogotá determinaremos dos clasificaciones:

- Problemáticas externas

- Problemáticas internas

Las pymes están expuestas a un ambiente de extrema competitividad, que está marcado por la entrada de nueva competencia; tanto nacional como extranjera, la inestabilidad financiera, exigencias de alta calidad por parte de los clientes y desarrollo de nuevas tecnologías.

Este entorno tan agresivo dificulta el crecimiento de las pequeñas y medianas empresas (Pymes) de la ciudad de Bogotá, por lo cual se hace necesario obtener un alto nivel de competitividad (al menos $80 \%$ ) que les permita a las pymes superar las principales problemáticas que las limitan y afianzarse en el mercado.

Las Pymes en Colombia generan cerca del 68\% del empleo del país, es necesario aclarar que en el país existen 2,5 millones de micro, pequeña y mediana empresa y que del total de estas el $66 \%$ se encuentran ubicadas en Bogotá según la cámara de comercio de Bogotá "Economia de la Region Bogotá-Cundinamarca" de 2016, en donde existen más de 370.000 empresas, las pymes se encuentran distribuidas de la siguiente forma en la ciudad: suba (19\%), Engativá (14\%) Usaquén (12\%) y Kennedy (11\%) y que constituyen el $86 \%$ de las empresas de Bogotá. Fig. 1.

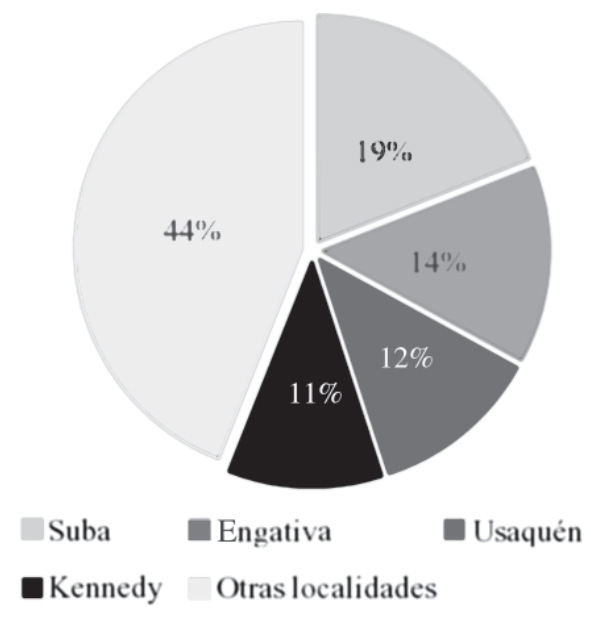

Fig. 1. Distribución de las Pymes en Bogotá.
Este estudio también ubica las pymes por sector: de estas el $62 \%$ de la pymes operan en el sector servicios, el $13 \%$ en el sector de las industrias culturales y creativas, el $6 \%$ en el sector manufacturero, el $5 \%$ en el sector agrícola o agroindustrial y el otro $10 \%$ en el sector confecciones. Solo un $4 \%$ se ubica en el sector del calzado y marroquinería, Entendiendo la numerosa cantidad de Pymes que existen en la ciudad ahora procederemos a identificar los problemas centrales que afrontan estas empresas e impiden el aumento de la competitividad desde la perspectiva del capital humano:

- Baja capacidad de administración.

- Dificultad para contratar empleados calificados.

- Subutilización de la capacidad de la instalación.

- Falta de liderazgo.

- Baja participación de los empleados.

- Dificultad para negociar con proveedores.

- Personalización del producto al cliente.

- Poco acceso a la información.

- Baja productividad y calidad de productos.

- Poca innovación.

- Producción reducida y poca flexibilidad.

Un gran reto para la empresa moderna según Hernández es: “Utilizar los recursos disponibles para conseguir la máxima efectividad y economía de los bienes y servicios que la gente necesita y desea. Para hacer frente a las demandas del mercado de consumo se requieren diferentes tipos de actividad empresarial" [1].

Y es ahí donde recae el problema principal de la mayoría de las pymes, es importante subrayar que la gran mayoría de limitantes que las Pymes poseen es consecuencia de las fallas en el conocimiento del empresario frente a la toma de decisiones y estrategias que la organización adopta para aumentar su competitividad. 


\section{Antecedentes}

Las Pymes del país dependen principalmente del mercado interno según "Economia de la region Bogotá- Cundinamarca", son pocas las empresas que pueden competir en un mercado globalizado y con la llegada de multinacionales al país las micro y pequeñas empresas enfrentan una situación desventajosa, esto ha obligado a las empresas a realizar modificaciones en sus estrategias y funcionamiento, además de los problemas tradicionales que afectan las Pymes del país [2].

El termino competitividad es muy utilizado y tienen mucha incidencia a la hora de iniciar y desarrollar cualquier iniciativa. Reta define la competitividad como la capacidad de cualquier organización para perseverar en forma ordenada y precisa ventajas comparativas que le permitan alcanzar y mantener una determinada posición en el entorno social y económico [3].

Por otro lado la competitividad empresarial se define como el cumplimiento de aspectos:

1. Innovación tecnológica y metodología de gestión.

2. Posicionamiento en el sector.

3. Eficiencia en los costos.

4. Utilización del capital humano.

Para que una empresa logre una distinción en el mercado, debe sin lugar a dudas generar una ventaja competitiva, la ventaja competitiva según Porter, menciona [4]:

- Diferenciación: significa que una firma intenta ser única en una industria en algunas dimensiones que son apreciadas por los compradores.

- Enfoque: significa que una organización fijo ser la mejor en un segmento o grupo de segmentos. Tabla 1.

Las personas deben ser competitivas para alcanzar sus objetivos y considera que el capital humano en la clave para alcanzar la competitividad institucional [5].
Tabla I. Cuadro Comparativo.

\begin{tabular}{|ll|}
\hline Ventaja competitiva & Ventaja comparativa \\
\hline Según Reta: es aquella & Según Porter: es aquella \\
ventaja que obtiene una & ventaja que goza un país \\
$\begin{array}{l}\text { organización cuando en } \\
\text { comparación con otro } \\
\text { produce con un menor } \\
\text { costo y con mejor calidad. }\end{array}$ & $\begin{array}{l}\text { cuanto al costo de } \\
\text { producción. }\end{array}$ \\
\hline
\end{tabular}

Fuente: Los autores.

El capital humano es aquel capital que generan todos los empleados de la organización y a través de sus motivaciones, capacidades y creatividad para generar un valor positivo que es capaz de influir en los resultados de la organización.

La teoría del capital humano ha sido desarrollada recientemente por el nobel de economía Amartya Sen, las aplicaciones de su propuesta donde define las capacidades que tienen los empleados para resolver las problemáticas empresariales se ha hecho a través de la teoría del capital humano [6].

Son reconocibles los aportes que el sector empresarial de las Pymes ha contribuido al desarro1lo, individual y colectivo de las personas y al haber asumido compromisos con el desarrollo personal y social de una comunidad, pero la teoría del capital humano propone en palabras de Schultz "al invertir en sí mismos, los seres humanos aumenta el campo de posibilidades [7]. Es un camino por el cual los hombres pueden aumentar su bienestar", también "Propongo tratar la educación como una inversión en el hombre y tratar sus consecuencias como una forma de capital. Como la educación viene a formar parte de la persona que la recibe, me referiré a ella como capital humano"[7].

La teoría del capital humano se enmarca dentro de los supuestos básicos:

- La existencia de un mercado de trabajo competitivo.

- Individualismo de las decisiones de las personas.

- Fácil acceso a las fuentes de financiación. 
Así mismo con el nacimiento de esta teoría han sido múltiples los aportes de autores que han contribuido a la creación de un concepto de capital humano [7].

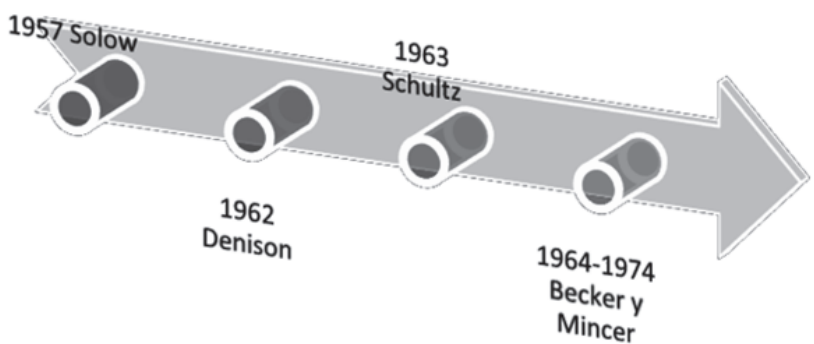

- Solow-1957: ser humano como componente fundamental en el desarrollo productivo de la industria.

- Denison-1962: personal mejor capacitado aumenta la calidad de la fuerza de trabajo.

- Schultz-1963: la calidad tiene un valor y se manifiesta por medio de la adquisición en capital humano.

- Becker-1664: los gastos de educación al mismo tiempo son un costo de oportunidad que en un futuro aumentara las ganancias de la organización y del empleado.

- Mincer-1974: el valor de la experiencia.

\section{Metodología}

En esta parte del proceso se realizará una revisión bibliográfica y se realizará una encuesta a dueños de pymes de Bogotá en el sector de Suba, Villamaria.

Se comienza una encuesta exploratoria a las pymes del sector deSuba villamaria, principalmente a empresas prestadoras de servicios y se les realiza la siguiente pregunta:

1. ¿Cuál cree usted que es el principal problema de las pymes para aumentar su competitividad con respecto al capital humano?

La encuesta arroja los siguientes resultados:

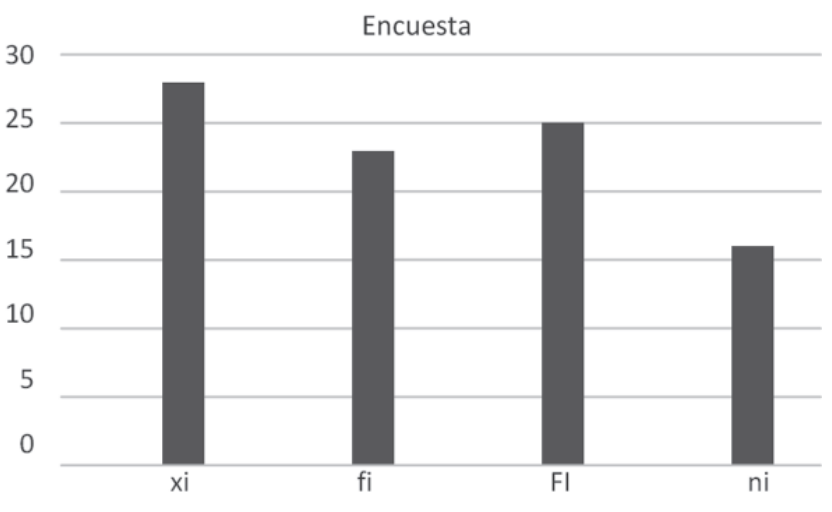

Tabla de frecuencia

\begin{tabular}{|lll|}
\hline & & NI \\
\hline xi & 28 & $56 \%$ \\
fi & 23 & $46 \%$ \\
FI & 25 & $50 \%$ \\
ni & 16 & $32 \%$ \\
\hline
\end{tabular}

Como se ve en la tabla:

$\mathrm{xi}=$ número de personas que contestaron que era la falta de crédito para capacitaciones.

fi = número de personas que contestaron que era gestión administrativa deficiente.

FI= número de personas que contestaron que era falta de asociación.

ni= número de personas que contestaron que era falta de apoyo gubernamental.

$\mathrm{NI}=$ frecuencia de respuesta.

En la tabla de frecuencias se muestra que son muchos dueños de pymes que creen que las principales problemáticas por falta de competitividad con respecto al capital humano vienen desde el apoyo que ofrece el gobierno a las pymes y muchos de ellos consideran que es necesaria una intervención por parte del estado hacia los trabajadores que le permitan a estas organizaciones aumentar su competitividad.

Como se ve en la frecuencia se observa que la falta de crédito y poco asociación de las pymes con entes que permitan a los trabajadores de las orga- 
nizaciones aumentar su nivel da capacitación es uno de los factores que más evidencias los empresarios de las pymes de los sectores prestadores de servicios y que ellos consideran son los más importantes para medir la competitividad del capital humano.

Realizando una revisión bibliográfica se identifican otras metodologías para aumentar la competitividad desde el capital humano, estas son:

1. Teoría de la motivación: La participación en el mercado es clave para la supervivencia de nuestras empresas locales, las cuáles deben preparar todas y cada una de las actividades y ajustarse a las necesidades y oportunidades de los mercados para llegar a ser participantes ya que debido a la globalización gradual del mercado, las empresas cada vez tienen menos tiempo para ajustarse a las nuevas realidades. Aunado a esto, están tan expuestas a la competencia desleal que deben participar competitivamente para poder generar mayores beneficios [8].

2. La inteligencia generadora de ideas: Hombres y mujeres con una inteligencia emocional desarrollada se comportan: sociales y alegres, con una notable capacidad de compromiso, siendo solidarios, expresando sentimientos abierta y adecuadamente y comunicándose en forma fluida [9].

3. Teoría del pensamiento creativo: Mientras se siga pensando que la creatividad es un don personal, las empresas están ignorando miles de ideas que laten entre sus empleados [10].

\section{Resultados ESPERADOS Y DISCUSIÓN}

Después de haber hablado de cuáles son las características y problemas que imperan a las pymes y conocer diferentes teorías que pueden influir positivamente en las pymes prestadores de servicio, resulta de vital importancia resaltar cuales serían las posibles alternativas que contribuyan a solucionar las problemáticas y ayuden a las pequeñas empresas a sobrevivir a aumentar su competitividad.
Estas alternativas son:

1) Establecer objetivos de innovación con los empleados.

2) Fomentar la capacitación continua de los empleados.

3) Realizar jornadas de charlas con los empleados.

4) Establecer procedimientos de ejecución y verificación de nivel de calidad del servicio.

5) Implementar sistemas de evaluación de las capacidades de los empleados de forma continua.

6) Evaluar loa efectividad y progresos de la capacitación de los empleados.

En necesario que los pequeños empresarios comprendan que la competitividad es el reto más impórtate que deben enfrentar, las Pymes prestadoras de servicio no pueden basar sus operaciones en el bajo costo de mano de obra y que una participación activa del capital humano tiene un nivel de innovación muy alto dentro de las organizaciones que ahora con el desarrollo de nuevas tecnólogas y la continua exigencia de los clientes por la alta calidad es constante.

Las pymes prestadoras de servicio de Bogotá solo tienen dos soluciones para aumentar su competitividad:

1. Involucrar al capital humano para que aporte ideas e innove de manera continua y así las pymes puedan alcanzar los objetivos que se proponen.

Para esto el empresario tiene que tener una gran visión y capacidad para seleccionar y entregar tareas a su equipo de trabajo, además de desarrollar en ellos la dedicación por el trabajo, el estudio, la responsabilidad y el compromiso para transmitirles la excelencia.

2. Buscar soluciones de asociación tecnología y unir el capital humano con maquinara de alta innovación, en esta opción la organización ya no dependerá de si misma sino que deberá apoyarse en las ideas de otros y tratar de 
explotar al máximo el capital humano, es importante resaltar que los cambios tecnológicos no deben realizarse sin estudios que soporten la inversión.

\section{CONClusiones}

Realizando la investigación bibliográfica se encuentra que aunque son diversas las problemáticas que afrontan las pymes que son de carácter externo y tienen una alta influencia del entorno, muchas veces las soluciones que ofrece el capital humano son suficientes para resolverlas.

El capital humano es la principal fuente de calidad de las pymes, son el soporte de la organización y la alternativa más viable para solucionar problemas.

El éxito de una pyme radica en la competitividad, para conseguir una alta competitividad que es la máxima utilización posible de sus recursos las pymes deben modernizar su maquinara y actualizar su capital humano, dado que su principal fortaleza es su simplicidad esta competitividad se alcanzara desde el capital humano.

En caso de aplicar alguna de las posibles soluciones en una Pyme del sector de servicios, tendrá un tiempo estimado de aplicación de entre 2 o 3 meses y un posible resultado de aumento de competitividad de aproximadamente un $30 \%$ manera casi inmediata.

\section{REFERENCIAS}

[1] Hernández. Ventaja competitiva. Creatividad para tu negocio, Pyme Adminístrate Hoy. 2008.

[2] Economía de la región Bogotá-Cundinamarca. Bogotá. 2016.

[3] M. Reta, Políticas para la Competitividad. México Pyme Hoy. México. 2008.

[4] Porter. "What is Strategy?". Harvard Business Review, 62-78. 1995.

[5] J. Saracho, Un Modelo General de Gestión por Competencias.Santiago de Chile, Chile: Ril Editores. 2005.

[6] M. Alcañiz Moscardó, El desarrollo local en el contexto de la globalización. Convergencia, 15(47), 285315. Recuperado en 05 de abril de 2019, de http:/ / www.scielo.org.mx/scielo.php?script=sci_arttext \&pid=S1405-14352008000200011\&lng=es\&tlng= es. 2008.

[7] SCHULTZ, Theodore W. (1985). "Investing in people. The economics of population quality». Págs. 9- 135. Editorial Ariel, S.A. Espan?a, diciembre 1985.

[8] Robbins, D. (1996).Fundamentos de Administración.México, DF, México: Pearson Hall.

[9] Gibson, J., Ivancevich, J. yDonnelly, J. (2001). Las Organizaciones (Comportamiento, estructura, procesos)(10ma Ed.). Santiago de Chile, Chile: Mc Graw Hill Interamericana.

[10] De Bono, E. (2000).El Pensamiento Lateral, Manual de creatividad.México, DF, México: Paidós Empresas. 